\title{
Strategy Decision of Business Interruption Insurance and Emergency Supply Strategy Based on Supply Disruptions
}

\author{
Li Xin-jun ${ }^{1,2}$, Wang Li-jie \\ ${ }^{1}$ School of Economic and Management, Yantai University (China) \\ ${ }^{2}$ Faculty of Management and Economics, Dalian University of Technology (China) \\ lixinjun101@,163.com,number1wli@,botmail.com
}

Received: December 2014

Accepted: February 2015

\section{Abstract:}

Purpose: Recent years have witnessed the pervasive supply disruptions and their impacts on supply chain performance. It is significant for enterprises to adopt comprehensive measures to cope with supply disruptions. The purpose of this study is to investigate how BI insurance makes up the shortage of emergency supply and affect the expected profit of enterprises.

Design/methodology: This study develops the penalty cost function on the basic of the financial costs caused by the interruption losses, introduces variables of BI insurance and operational measures, establishes the profit model with BI insurance or not.

Findings: Through the proof and analysis, it is demonstrated that BI insurance can mitigate the adverse effect of the increasing cost for expected profit. And this study finds that the value of $\mathrm{BI}$ insurance is higher when interruption probability is lower and penalty coefficient is higher.

Originality/value: In this study, it is investigated that the impact of business interruption (BI) insurance on supply disruptions and its complementary value against the higher purchase cost of emergency sourcing strategy. BI insurance is an efficient measure for supply interruption and should be adopted correctly to play a role in managing supply disruption risk.

Keywords: supply disruptions, emergency supply, business interruption insurance, risk management 


\section{Introduction}

With the development of economic globalization, supply chains have become highly complex under uncertainties of the operating environment and vulnerability of supply system. Supply disruptions could bring the huge interruption loss to the buyer, so he should take integrated and effective measures to cope with supply disruptions. Unprecedented supply disruptions of Shanghai FuXi event brought a huge loss to the multinational chain restaurant enterprises, e.g. KFC and McDonald.

To mitigate supply disruptions, buyers may hold emergency inventory, source form multiple suppliers, and emergency purchase form backup suppliers. Business interruption insurance (BI) is more and more favored and has played a huge role in reducing the loss of supply interruption. BI insurance, as is a relatively passive cash compensation measure, must be combined with other proactive and initiative emergency operational measures. Due to supply disruptions of Philips mobile phone chip in 2000, Ericsson received $\$ 20$ million from business interruption insurance, and Nokia promptly took another active emergency supply measure. Although Ericsson obtained insurance compensations, it lost a lot of market share because of passively waiting, while Nokia increased a lot of market share by taking positive measures. Business interruption insurance can play a greater role if it is combined with other effective operational measures.

The development of business interruption insurance is seriously lagging behind in our country and the application is also more slowly in supply chain in virtue of the inadequate promotion of insurance company and insufficient awareness of enterprises. Companies tend to adopt separately emergency inventory or emergency backup supplier, while they ignore business interruption insurance.

In this paper, the complementary role of business interruption insurance for operational measure and its value are explored. It will enhance the importance of manufacturer for BI insurance. The integrated measure can play a more effective role when business interruption insurance and emergency measures are combined.

\section{Literature Review}

There has been a growing stream of literature on supply disruptions. Tomlin (2009) investigated two supply strategies of supplier diversity and emergency back-up supply strategy. Li, Ji and Wang (2014) explored dual-sourcing strategies and coordination mechanism of rewards \&punishments mechanism and revenue-sharing contract to mitigate disruption risk, dividing disruptions into common cause failure and supplier-specific incident. Tomlin (2006) considered the case of adopting two suppliers with different cost and reliability. Dada, Petruzzi and Schwarz (2007) constructed the distribution newsboy orders and supplier selection problem. Zhu and Fu (2013) explored the trade-off between ordering policies and disruption risks in a dual-sourcing network under specific (or not) service level constraints. Babich, 
Burnetas and Ritchken (2007) investigated the decision of pricing, ordering and vendor selection under random interruption.

Our study is also related to the literature on BI insurance. Kuzak and Larsen (2005) proposed insurance pricing way including risk factors: premiums equal to the long-term average compensation plus cost burden plus burden of risk. Tao and Geng (2008) illustrated calculated evidence of the insurance amount and premium pricing way in America and British. Xu (2007) studied the vendor extensions liability of the insured production relies on a dedicated or a few specialized suppliers of raw materials. Mao (2008) analyzed the differences of expansion of business interruption insurance liability in our and foreign country.

Our study is also closely related to extensive literature on interruption risk management based on BI insurance. Tomlin and Dong (2012) introduced BI insurance to the enterprise risk management for the first time. They established profits model of manufacturers on the basis of unlimited interruption time, considered the S-type (inventory not damaged) and the F-type interrupt type. Cai (2013) analyzed the impact of business interruption insurance on inventory levels with a fixed downtime, interruption probability obey Bernoulli distribution. Zhao (2013) established BI insurance purchase profit model in both decentralized and centralized case based on discrete model in finite time.

Most of these documents presented emergency measures but literature about supply interruption risk based on BI insurance is rare. Some literatures only investigate the influence of BI insurance on emergency stocks without analyzing its impact on other supply emergency measures. In this article, we introduce business interruption insurance into supply disruptions. Profit model is built with the integration of inventory, emergency procurement and BI insurance based on classic stock and emergency supply strategy. We analyze complementary role of BI insurance for emergency procurement measure and its contribution to the expected profit, in addition we explore penalty cost function and the value of BI insurance.

\section{Model Assumptions and Symbol Description}

\subsection{Model Assumptions}

We model a supply chain consisting of a supplier and an emergency supplier, and a manufacturer. The manufacturer purchase BI insurance and obtain compensation when supplier interrupts. We consider the manufacturer adopts a basic-stock policy in which the beginning inventory is brought to a level $B$ in any $n$ period. When supply disruptions occur, the manufacturer firstly produces using the remaining inventory.

The manufacturer suffers loss of income due to interruption and thus falls into tangible cash shortages, and it will give rise to the cost of external financing and the losses of external investment income, which we call penalty cost represented by the penalty cost function $F(I L)$. BI insurance not only gains tangible compensations but also makes benefits by avoiding 
penalty costs, and we use compensation coefficient to describe this part of compensation. The punishment cost function is a linear function of interruption losses. The whole operation process is shown in Figure 1. Other assumptions are as follows.

- Supplier delivery stock of $B$ regularly and supply nothing under disruption.

- Manufacture pays a reservation fee $b$ every $n$ period to ensure emergency supplier availability, and the executive costs $e$ with $c<e<r$.

- In order to simplify the model, we assume supplier can only disrupt once within the BI insurance period.

- The basic inventory $B$ is valued by the time it can maintain regular production, and $n$ $<B<2 n$.

- If the downtime is less than the time stock $B$ can last, it will not bring interruption losses, so we assume the interruption time $t>B$, manufacture needs to take emergency supply measure when inventory downs to zero.

$$
\text { No interruption Interruption stage Recover supplying }
$$

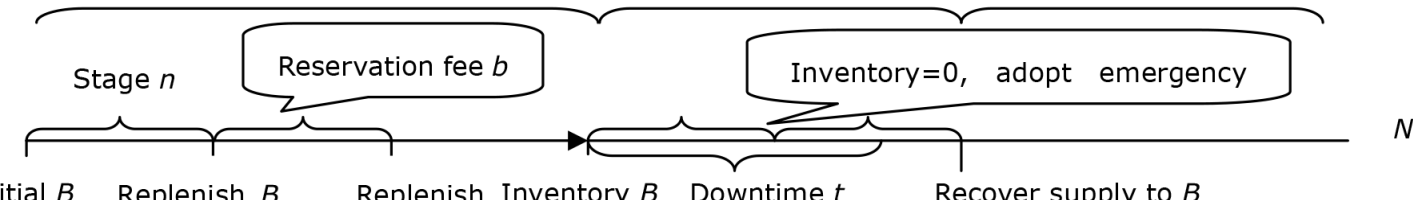

Initial $B \quad$ Replenish $B \quad$ Replenish Inventory $B$ Downtime $t \quad$ Recover supply to $B$

Figure 1. Operation course based on BI insurance and emergency supply

\subsection{Model Parameters}

Model parameters are shown in Table 1.

\begin{tabular}{|c|l|c|l|}
\hline Parameter & \multicolumn{1}{|c|}{ Definition } & Parameter & \multicolumn{1}{|c|}{ Definition } \\
\hline$r$ & Revenue per unit of finished product & $I L$ & Tangible profits loss in interruption \\
\hline$c$ & Cost per unit of raw material & $F(I L)$ & Penalty cost function: $F(I L)=k(I L), k>0$ \\
\hline$e$ & Emergency sourcing cost per unit $(c<e<r)$ & $t$ & Interruption duration \\
\hline$b$ & Reservation fee per period & $a$ & Premium rates \\
\hline$v$ & Variable production cost per unit & $\beta$ & Insurance compensation rates \\
\hline$f$ & Fixed cost per period & $\gamma$ & Rate of penalty cost compensation \\
\hline$g$ & Goodwill cost per unit lost sale & $\Pi^{E}$ & Expected profit without BI insurance \\
\hline$h$ & Inventory holding cost per unit & $\Pi_{l}^{E}$ & Expected profit with BI insurance \\
\hline$B$ & Basic inventory level & $\pi_{e l}^{D}$ & Profit per unit under interruption \\
\hline$n$ & Order cycle & $N$ & BI insurance coverage period \\
\hline
\end{tabular}

Table 1. Parameters definition 


\section{Emergency Management Model Based on BI Insurance}

\subsection{The Basic Model}

The manufacture's profit within $n$ stage can be written as follows when supply disruption does not occur.

$$
\pi_{e}^{U}=n(r-v)-f-1 / 2(B+B-n) \times n \times h-b
$$

The manufacturer gains the following profit for the period $t$ without BI insurance under supply disruption,

$$
\pi_{e}^{D}=B(r-v)+(t-B)(r-e)-(t / n) b-(t / n) f-1 / 2 B \times B \times h
$$

Therefore, without BI insurance within the insured period $N$, the expect profit is given by

$$
\Pi^{E}=N\left[P(A) \pi_{e 1}^{U}+P(B) \pi_{e 1}^{D}\right]-P(B) F\left(I L_{E}\right)
$$

where $P(B) F\left(I L_{E}\right)$ is comprehensive penalty cost caused by interruption losses. When interruption does not occur, the manufacture's profit per unit time $\pi_{e 1}^{U}$ is

$$
\pi_{e 1}^{U}=[n(r-v)-f-1 / 2(B+B-n) n h-b] / n
$$

When interruption occurs, the manufacture's profit per unit time $\pi_{e 1}^{D}$ is

$$
\pi_{e .1}^{D}=[B(r-v)+(2 n-B)(r-e)-2 b-2 f-1 / 2(B \times B \times h)] / 2 n
$$

According to the related criteria of BI insurance, it is only responsible for tangible interruption losses, not for intangible losses. Therefore, only tangible profits loss $I L$ is insured, except for loss of goodwill and penalty costs. The loss function of a tangible profit within an insurance cycle is

$$
I L_{E}=N P(B)\left(\pi_{e l}^{U}-\pi_{e l}^{D}\right)=N P(B) \frac{(2 n-B)(e-v)-[(2 B-n) n h-1 / 2 B B h]}{2 n}
$$

The manufacturer's profit $\prod_{l}^{E}$ with $\mathrm{BI}$ insurance is

$$
\prod_{l}^{E}=\prod^{E}+I L_{E}(\beta P(B)-\alpha)+\gamma F\left(I L_{E}\right)
$$

Where $\Pi^{E}$ is the expected profit without BI insurance. Premium rate $a$ is the ratio of the price the manufacturer pays the insurer to the insurance coverage. Insurance compensation rates $\beta$ is the ratio of the compensation to the insurance coverage. Rate of penalty cost compensation $Y$ is compensation ratio of comprehensive penalty costs with BI insurance, with $0<y<1$.

The effect of BI insurance is determined by the net premiums and net benefit. Premiums is a fixed value being paid in advance as sunk costs, while the insurance compensation exits only in the event of interruption loss i.e. $\beta P(B)-\alpha<0$. We specify $I L_{E}(\beta P(B)-\alpha)$ as the net premium 
and $Y F\left(I L_{E}\right)$ as the net income in addition to the average compensation. BI insurance can increase the expected profits of the manufacturer when the gaining net income is greater than the paying net premiums, i.e. $(\beta P(B)-\alpha)+\gamma k>0$

\subsection{Interaction between BI Insurance and Emergency Supply}

Theorem 1: The manufacturer's expected profit decreases as emergency procurement cost increases, but BI insurance can slow down the decrease, and the greater the emergency procurement cost is, the trend of slowing down is more obvious.

Proof: Substituting formula (4) and (5) into formula (3), we can obtain expected profit function without BI insurance, taking a first-order derivative with respect to $e$

$$
\frac{\partial \prod^{E}}{\partial e}=N P(B)\left(-1+\frac{B}{2 n}\right)+N P(B)\left(1-\frac{B}{2 n}\right) \times k=N P(B)\left(1-\frac{B}{2 n}\right) \times(k-1)
$$

Expected profit without BI insurance decreases with the increase in emergency procurement costs and booking fees because formula ( 8 ) is less than zero.

Put formula (3) and (6) into formula (7) to obtain expected profit function with BI insurance,

$$
\begin{aligned}
& \prod_{l}^{E}=\prod^{E}+I L_{E}(\beta P(B)-\alpha)+\gamma F\left(I L_{E}\right) \\
& =\prod^{E}+N P(B)\left(\pi_{e 1}^{U}-\pi_{e 1}^{D}\right)[(\beta P(B)-\alpha)+\gamma k] \\
& =\prod^{E}+N P(B)[(\beta P(B)-\alpha)+\gamma k] \times \frac{(2 n-B)(e-v)-[(2 B-n) n h-1 / 2 B B h]}{2 n}
\end{aligned}
$$

From a first-order derivative of (9) to $e$, we have

$$
\begin{aligned}
& \frac{\partial \prod_{l}^{E}}{\partial e}=N P(B)\left(1-\frac{B}{2 n}\right)(k-1)+N P(B)[(\beta P(B)-\alpha)+\gamma K] \times \frac{2 n-B}{2 n} \\
& =N P(B)\left(1-\frac{B}{2 n}\right)[(k-1)+(\beta P(B)-\alpha)+\not k]
\end{aligned}
$$

F or $N P(B)\left(1-\frac{B}{2 n}\right)>0, \beta P(B)-\alpha<0(k-1)+\not k<0$, therefore $\frac{\partial \prod_{l}^{E}}{\partial e}<0$, expected profit decreases with the increase of emergency procurement costs e with BI insurance.

To obtain the profit trend with the emergency procurement costs with BI insurance or not, we use formula (3) minus formula (9).

$$
\begin{aligned}
& \prod_{l}^{E}-\Pi^{E}=N P(B)\left(\pi_{e 1}^{U}-\pi_{e 1}^{D}\right) \times(\beta P(B)-\alpha)+\gamma \times k \times N P(B)\left(\pi_{e 1}^{U}-\pi_{e 1}^{D}\right) \\
& =N P(B) \times \frac{(2 n-B)(e-v)-[(2 B-n) n h-1 / 2 B B h]}{2 n}(\not k+\beta P(B)-\alpha)
\end{aligned}
$$


The first derivative of (11) to $e$ is given as,

$$
\left.\frac{\partial\left(\prod_{l}^{E}-\Pi^{E}\right)}{\partial e}=N P(B)\left(1-\frac{B}{2 n}\right)[(\beta P(B)-\alpha)+\gamma k)\right]
$$

For $N P(B)\left(1-\frac{B}{2 n}\right)>0$, therefore when $(\beta P(B)-\alpha)+\gamma k>0$, formula (12) is greater than 0.The margin profit of $\mathrm{BI}$ insurance or not increases with $e$ when the value of $\mathrm{BI}$ insurance is positive. In other words, BI insurance reduces the probability of giving up emergency supplier. The adoption of BI insurance can make up the limits of emergency supply. In fact, emergency supplier price under supply disruptions is higher than usual, BI insurance mitigates negative impact of increased price on corporate profits. BI insurance and emergency supply strategy interact and promote each other, jointly facilitating to minimize interruption losses.

\subsection{Operation Mechanism of BI Insurance}

Theorem 2: The expected profit with BI insurance is a convex function of interruption probability. The effect of BI insurance highlights when the interruption probability is relatively lower; the effect of inventory and emergency procurement highlight when the interruption probability is larger and exceeds the critical.

Proof: Expected profit function with BI insurance is given by

$$
\left.\prod_{I}^{E}=N\left[P(A) \pi_{e 1}^{U}+P(B) \pi_{e 1}^{D}\right]-P(B) F\left(I L_{E}\right)+N P(B)[\beta P(B)-\alpha)+\gamma k\right]\left(\pi_{e 1}^{U}-\pi_{e 1}^{D}\right)
$$

From first-order and second-order derivative of $(13)$ to $P(B)$, we have

$$
\begin{aligned}
& \frac{\partial \prod_{l}^{E}}{\partial P(B)}=N \pi_{e 1}^{D}-2 N P(B)\left(\pi_{e 1}^{U}-\pi_{e 1}^{D}\right)+[2 N(P(B) \beta-\alpha)+\gamma k]\left(\pi_{e 1}^{U}-\pi_{e 1}^{D}\right) \\
& \frac{\partial^{2} \prod_{l}^{E}}{\partial(P(B))^{2}}=-2 N\left(\pi_{e 1}^{U}-\pi_{e 1}^{D}\right)+2 N \beta\left(\pi_{e 1}^{U}-\pi_{e 1}^{D}\right)=2 N(\beta-1)\left(\pi_{e 1}^{U}-\pi_{e 1}^{D}\right)<0
\end{aligned}
$$

It appears that the profit increases firstly and then decreases with the probability within an insurance period because expected profit function is a convex function of interruption probability. When the interruption probability is lower, BI insurance has effect on tangible compensation and avoidance of punishment costs. When the interruption probability exceeds a certain value, the manufacturer cannot get more compensation due to the presence of maximum insured limits, necessary to adopt inventory policy and emergency supply strategy. Therefore, the benefit of BI insurance is larger when interruption duration is relatively shorter; otherwise, manufacturer should timely adopt emergency supply strategy and other positive operational measures when interruption duration is relatively longer. 
Theorem 3: The effect of BI insurance is larger when manufacturer has less own funds or more external investment opportunities, otherwise the effect is smaller.

Proof: Putting formula (3) and (6) into formula (8), we have

$$
\prod_{l}^{E}=N\left\lfloor P(A) \pi_{e 1}^{U}+P(B) \pi_{e 1}^{D}\right\rfloor-N P^{2}(B) k\left(\pi_{e 1}^{U}-\pi_{e 1}^{D}\right)+N P(B)[\beta P(B)-\alpha+\gamma k]\left(\pi_{e 1}^{U}-\pi_{e 1}^{D}\right)
$$

Taking first-order derivative to $k$, we have

$$
\frac{\partial \prod_{l}^{E}}{\partial k}=N P(B)\left(\pi_{e 1}^{U}-\pi_{e 1}^{D}\right)(P(B)+\gamma)>0
$$

Expected profit with BI insurance increases as the penalty coefficient $k$ increases. The interruption leads to greater financial costs (penalty costs) when manufacturer owns less funds or more external investment opportunities, then BI insurance has larger value for manufacturer. In this case, manufacturer should purchase BI insurance to avoid penalty cost efficiently and to prove expected profit.

Business interruption insurance as an effective measure for supply disruptions plays a role in monetary compensation, penalty cost avoidance and making up for the limitations of operational measures. But as a financial tool, BI insurance also has its own limits. The provision of maximum coverage limits makes manufacturer cannot obtain the corresponding compensation for damage, which also determines that BI insurance is not a positive and longterm significant measure. The value of BI insurance reduces when interruption loss is more than the specified maximum insured amount. In practice, manufacturer should select inventory, emergency supply and other positive operation strategies to deal with supply interruption. BI insurance should be used as an aid policy so as to achieve the purpose of reducing interruption losses.

\section{Analysis of Numerical Example}

This section provides in-depth analysis of the interaction mechanism of BI insurance and emergency supply strategy, exploring its impact on expected profits. Parameter assignments are shown in Table 2.

\begin{tabular}{|c|c|c|c|c|c|c|c|c|c|c|c|}
\hline $\boldsymbol{B}$ & $\boldsymbol{N}$ & $\boldsymbol{n}$ & $\boldsymbol{f}$ & $\boldsymbol{v}$ & $\boldsymbol{h}$ & $\boldsymbol{c}$ & $\boldsymbol{r}$ & $\boldsymbol{a}$ & $\boldsymbol{\beta}$ & $\boldsymbol{\gamma}$ & $\boldsymbol{k}$ \\
\hline 45 & 360 & 30 & 20 & 0.1 & 0.006 & 0.8 & 4 & 0.05 & 0.8 & 0.8 & 0.5 \\
\hline
\end{tabular}

Table 2. Parameter assignments 


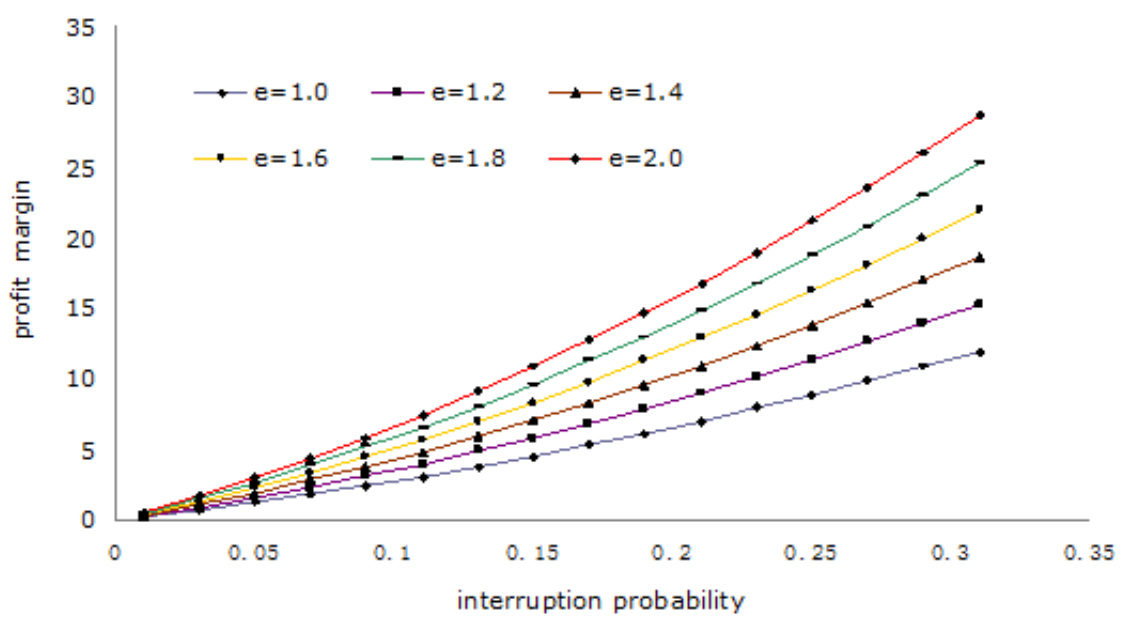

Figure 2. Impact of BI insurance on emergency supply measures

Using formula (11), assignment for e according to assumptions on the basis of assignment of each variable parameters above in Table 2 and calculate the respective profit difference, we get Figure 2. Through observation and analysis, we can get the conclusions as follows: The expected profit with insurance is more than profit without insurance within a reasonable range of interruption probability and the profit difference is positive; The profit margin increases with given interruption probability, it can be inferred that the effect of purchasing BI insurance increases with $P(B)$ before reaching the critical and this conclusion is correspond to the above prove of theorem 2; The curve of $e=1.0$ is flatter, while the curve of $e=2.0$ is steeper, so we can infer that the growth trend of profit margin is more obvious and the effect of BI insurance is larger when cost $e$ is higher; In addition, profit margin increases with the increased emergency procurement cost $e$ at the same level of interruption probability.

Penalty coefficient $k$ depends on company's financial situation, such as financing and investment conditions. $k$ influences the value of $\mathrm{BI}$ insurance in the different cases. Manufacturer should judge the value of BI insurance according to their own financial situation and make the right decisions. Following we let booking fee $b=30$, emergency procurement cost $e=1.6$, and calculate respective expected profit in $k[1,4]$. Through observation and analysis to Figure 3, we can get the conclusions: The curve of $k=1$ is flatter and the curve of $k=4$ is steeper, it can be inferred that the profit with BI insurance increases more rapidly with the interruption probability when $k$ is larger before exceeding the critical. Expected profit with BI insurance increases with the penalty coefficient $k$ increases, thus the value of BI insurance increases. 


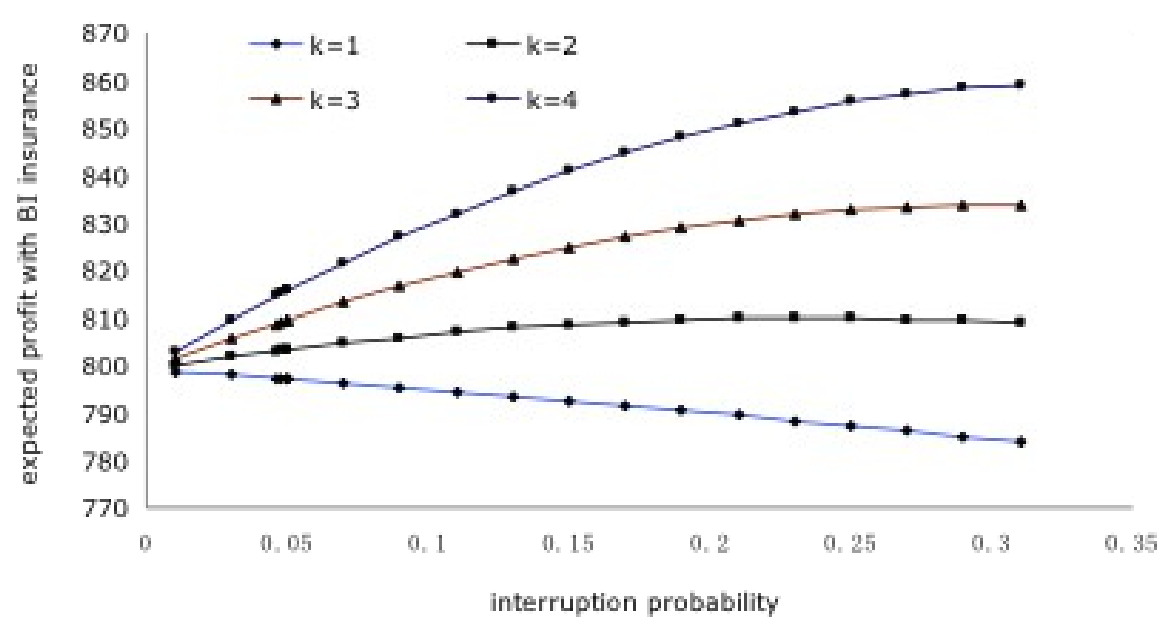

Figure 3. Analysis of BI insurance value with different $k$

\section{Conclusions}

In this paper, we investigate the complementary role of BI insurance for emergency supply measure. BI insurance reduces negative impact of increased emergency procurement cost and makes up for the limits of emergency supply strategy. This paper supplements for the previous research of the influence of BI insurance on inventory and illustrates the complementary roles of BI insurance for operational measures. The value of BI insurance is greater when interruption probability is lower and interruption duration is shorter. The losses caused by interruption are greater than the maximum insured limits if interruption occurs with high probability or the duration is longer. In this case, the insurance company cannot adequately compensate for the interruption losses, then the value of the BI insurance and inventory strategy is declined while the value of emergency supply strategy highlights. In addition, manufacturer should also consider the financial situation to judge the value of BI insurance. The benefit of BI insurance is larger when manufacturer owns fewer funds or more external investment opportunities. In this paper, we assume that there is only one emergency supplier, but manufacturer often uses two or more emergency suppliers to cope with supply disruptions. Multiple vendors have different emergency procurement costs. Exploring the complementary role of $\mathrm{BI}$ insurance and its value in the case of adopting two emergency suppliers is a subject for future research. 


\section{Acknowledgments}

This work is supported by the National Social Science Foundation of China under Grant No. 12CGL042, the National Natural Science Foundation of China under Grant No. 71372122 and 71272122, and Shandong Natural Science Foundation under Grant No. ZR2010GQ005 and ZR2011GL019. The authors also gratefully acknowledge the helpful comments and suggestions of the reviewers, which have improved the presentation.

\section{References}

Babich, V., Burnetas, A.N., \& Ritchken, P.H. (2007). Competition and diversification effects in supply chains with supplier default risk. Manufacturing Service Operation Management, 2, 123-146. http://dx.doi.org/10.1287/msom.1060.0122

Cai, P. (2013). Risk Management research on supply interruption based on BI insurance. Journal of Guizhou University (Natural Sciences), 1, 36-38.

Dada, M., Petruzzi, N.C., \& Schwarz, L.B.(2007). A newsvendor's procurement problem when suppliers are unreliable. Manufacturing Service Operation Management, 1, 9-32. http://dx.doi.org/10.1287/msom.1060.0128

Kuzak, D., \& Larsen, T. (2005). Use of catastrophe models in insurance rate making. In eds. Grossi P, Kunreuther H. Catastrophe Modeling: A New Approach to Managing Risk, Springer US, 25, 97-118.

Li, X.J., Ji, J.H., \& Wang S.Y. (2014). Coordination and optimization of dual sourcing under supply disruptions. Journal of Industrial Engineering and Engineering Management, 3, 141-147.

Mao, X.N. (2008). Comparison of Chinese and foreign business interruption insurance extended responsibility. Shanghai Insurance, 4, 18-20.

Tao, C.W., \& Geng, Y.T. (2008). Business interruption insurance in foreign countries and its indication. Insurance Studies, 4, 6-10.

Tomlin, B. (2006). On the value of mitigation and contingency strategies for managing supply chain disruption risks. Management Science, 5, 639-657. http://dx.doi.org/10.1287/mnsc.1060.0515

Tomlin B. (2009). Disruption-management strategies for short life-cycle products. Naval Research Logistics, 4, 318-347. http://dx.doi.org/10.1002/nav.20344

Tomlin, B., \& Dong, L.X. (2012). Managing disruption risk: The interplay between operations and insurance. Management Science, 10, 1898-1915. http://dx.doi.org/10.1287/mnsc.1120.1524

Xu, C.M. (2007). Business interruption insurance. Shanghai: Fudan University Press.

Zhao, J.H. (2013). Research on supply chain risk management strategies based on the business interruption insurance. Doctoral Dissertation of Shanghai Jiaotong University. 
Zhu, J.J., \& Fu, S.C. (2013). Ordering policies for a dual sourcing supply chain with disruption risks. Journal of Industrial Engineering and Management, 1, 380-399.

http://dx.doi.org/10.3926/jiem.690

Journal of Industrial Engineering and Management, 2015 (www.jiem.org)

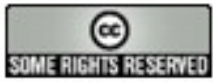

Article's contents are provided on a Attribution-Non Commercial 3.0 Creative commons license. Readers are allowed to copy, distribute and communicate article's contents, provided the author's and Journal of Industrial Engineering and Management's names are included. It must not be used for commercial purposes. To see the complete license contents, please visit http://creativecommons.org/licenses/by-nc/3.0/. 